\title{
PHOTOSYNTHESIS AND COLD ACCLIMATION: MOLECULAR EVIDENCE FROM A POLAR DIATOM ${ }^{1}$
}

\author{
Thomas Mock ${ }^{2}$ and Klaus Valentin \\ Alfred-Wegener-Institute for Polar and Marine Research, Am Handelshafen 12, 27570 Bremerhaven, Germany
}

The psychrophilic diatom Fragilariopsis cylindrus (Grunow) Krieger in Helmcke \& Krieger was used to investigate photosynthesis and growth under freezing temperatures. Gene expression during a temperature shift from $+5^{\circ} \mathrm{C}$ to $-1.8^{\circ} \mathrm{C}$ was studied under 3 and $35 \mu \mathrm{mol}$ photons $\cdot \mathrm{m}^{-2} \cdot \mathrm{s}^{-1}$ by using a macroarray. These measurements were paralleled by determination of fluorescence induction at PSII and pigment analysis. The shift to $-1.8^{\circ} \mathrm{C}$ at $35 \mu \mathrm{mol}$ photons $\cdot \mathrm{m}^{-2} \cdot \mathrm{s}^{-1}$ caused a marginal decrease of photosynthetic quantum yield $\left(\mathbf{F}_{\mathrm{v}} / \mathbf{F}_{\mathrm{m}}\right)$ from 0.61 to 0.52 with fast recovery after 1 day. The ratio of chl $c$ to $\operatorname{chl} a$ increased from 3.1 to 5.5, and the ratio of diatoxanthin to diadinoxanthin increased from 0.7 to 5.0. Genes encoding proteins of PSII $(p s b A, p s b C)$ and for carbon fixation $(r b c \mathrm{~L})$ were down-regulated, whereas genes encoding chaperons $(h s p 70)$ and genes for plastid protein synthesis and turnover (elongation factor $E f T s$, ribosomal protein $r p S 4, f t s H$ protease) were up-regulated. In contrast, cold exposure at $3 \mu \mathrm{mol}$ photons . $\mathrm{m}^{-2} \cdot \mathrm{s}^{-1}$ induced a marginal increase in $F_{v} / F_{m}$ from 0.61 to 0.63 and a strong increase in fucoxanthin concentrations from 0.04 up to $0.12 \mathrm{pg} \cdot$ cell $^{-1}$. This was paralleled by up-regulation of $f c p$ genes. The ratio of $\operatorname{chl} c$ to $\operatorname{chl} a$ also increased from 3.1 to 4.2 , as did the ratio of diatoxanthin to diadinoxanthin from 0.7 to 2.2. Down-regulation of $p s b A$, $p s b C$, and $r b c L$ could also be measured but not up-regulation of $h s p 70, E f T s, r p S 4$, and the $f t s H$ protease. The latter genes are probably necessary to avoid cold shock photoinhibition only at higher light intensities.

Key index words: cold acclimation; fluorescence induction; Fragilariopsis cylindrus; gene expression; macroarray; photosynthesis; pigments; sea ice

Abbreviations: DD, diadinoxanthin; DT, diatoxanthin; EST, expressed sequence tag; $\mathbf{F}_{v} / F_{m}$, quantum yield; $Q_{A}$, quinone $A$, first stable electron acceptor of PSII; $Q_{B}$, quinone B, second stable electron acceptor

The molecular mechanisms of acclimation and regulation of photosynthesis in psychrophilic polar dia-

\footnotetext{
${ }^{1}$ Received 15 December 2003. Accepted 14 April 2004.

${ }^{2}$ Author for correspondence: e-mail tmock@awi-bremerhaven.de.
}

toms are not well understood (Thomas and Dieckmann 2002). Most psychrophilic polar diatoms are able to live in a temperature range between +8 and $-1.8^{\circ} \mathrm{C}$, the freezing point of seawater (Fiala and Oriol 1990). In situ measurements in sea ice have shown that some of these diatoms are even able to grow at temperatures of $-15^{\circ} \mathrm{C}$, high salinities, and extreme light limitation (Mock and Gradinger 1999). However, most polar diatoms live in open water and at the sea ice-water interface where temperatures range from approximately +5 to $-1.8^{\circ} \mathrm{C}$.

Our knowledge of these globally important polar algae is mainly based on field and ecophysiological investigations with a limited potential to explain molecular mechanisms of photosynthesis regulation. Many interesting studies on polar diatoms have been conducted to reveal the main environmental factors that influence photosynthesis and growth. Polar diatoms are subject to a diverse number of abiotic stress factors, particularly low temperatures, freezing, high and low irradiance, and nutrient depletion (Kirst and Wiencke 1995, Boyd 2002). Nevertheless, no specific or unique "mechanism" inherent to polar diatoms has been detected to date. The physiology and metabolism of temperate and polar species do not seem to differ very much. Probably the key to understanding why polar diatoms are able to grow in polar waters and sea ice and why many of them are low light adapted must lie in their genomes. Genomes of some strains of temperate phytoplankton have now been completely sequenced, revealing their genetic adaptations to distinct marine niches (Dufresne et al. 2003, Fuhrman 2003, Rocap et al. 2003). These adaptations could not have been identified by using physiological measurements only. The combination of physiological and genomic approaches, however, has been more successful.

Recently, the U.S. National Academy of Sciences mapped out its strategy for advancing the understanding of polar organisms (Hoag 2003). Its report puts genomic sequencing and expression studies firmly at the center of this endeavor. We have already constructed the first expressed sequence tag (EST) library for the ecologically important polar microalga, Fragilariopsis cylindrus. This psychrophilic diatom is one of the most widely spread cold water diatoms and occurs in both the Arctic and Antarctic. It is regularly recorded from sea ice (Grunow 1884, Booth 1984, Okolodkov 1992, Gleitz et al. 1998) and from sea ice melt ponds (von Quillfeldt 1997) and may be one of the main 
biomass producers in polar plankton (Halldal 1953, Paasche 1960). Such a widespread occurrence within different polar marine habitats is probably related to the plasticity of its genome in the context of psychrophile.

We assume that photosynthesis in this diatom is probably not fundamentally different from that in other autotrophic organisms with respect to the presence of two photosystems (PSI and PSII), an electron transport chain, and RUBISCO as the primary $\mathrm{CO}_{2}$-fixing enzyme. However, there must be supplementary, still unknown, mechanisms to maintain photosynthesis and simultaneously protect the photosynthetic machinery against light damage, often observed in temperate plants and algae under low temperature conditions (Lyons 1973, Gombos et al. 1994, Jeong et al. 2002). Morgan-Kiss and colleagues, for instance, revealed such supplementary mechanisms in the Antarctic psychrophile Chlamydomonas subcaudata which has a different fatty acid composition in chloroplast lipid classes (Morgan-Kiss et al. 2002b) and is deficient in state I-state II transitions (Morgan-Kiss et al. 2002a).

Our experimental setup was chosen to analyze the expression of genes related to photosynthesis and cold acclimation during a temperature shift from the optimal temperature for growth $\left(+5^{\circ} \mathrm{C}\right)$ to the freezing point of seawater at two different irradiances. We used a subset of genes from the EST library and cloned additional relevant genes, which were combined on a macroarray. Gene expression studies were paralleled by biophysical and biochemical measurements relevant for photosynthesis.

\section{MATERIALS AND METHODS}

Algal material and growth conditions. Fragilariopsis cylindrus was collected from sea ice of the Weddell Sea (Antarctica) during the RV Polarstern expedition ANT XVI/3 from 18 March to 10 May 1999. After melting the sea ice in $0.2 \mu \mathrm{m}$ prefiltered seawater to a final dilution of $4: 1$ (volume seawater:volume sea ice), single cells were picked to obtain several clones of F. cylindrus. A well-growing clone was used for the cold exposure experiment. Cells were grown in light thermostats (Rumed-Thermostat type 1301, equipped with daylight fluorescence tubes, temperature accuracy $=$ max. $\pm 0.5^{\circ} \mathrm{C}$, Rubarth Apparate $\mathrm{GmbH}$, Hannover, Germany) in double F medium (Guillard and Ryther 1962) in 5-L batch cultures under optimum conditions defined as follows: growth rate $\left(0.72\right.$ division $\left.\cdot \mathrm{d}^{-1}\right)$ and maximum photochemical efficiency $\left(\mathrm{F}_{\mathrm{v}} / \mathrm{F}_{\mathrm{m}}=0.63\right)\left(+5^{\circ} \mathrm{C}, 35 \mu \mathrm{mol}\right.$ photons . $\mathrm{m}^{-2} \cdot \mathrm{s}^{-1}$ with a 16:8-h light:dark cycle). Bubbling with $0.2 \mu \mathrm{m}$ prefiltered air $\left(150 \mathrm{~mL} \cdot \mathrm{min}^{-1}\right)$ ensured sufficient $\mathrm{CO}_{2}$ supply and continuous mixing.

Six of these 5-L cultures were established, and the algae were left to grow under the same conditions until the beginning of the exponential growth phase. Subsequently, two replicates were chilled to the freezing temperature of seawater (approximately $-1.8^{\circ} \mathrm{C}$; only very few ice crystals could be detected in the flasks) at unchanged irradiance $(35 \mu \mathrm{mol}$ photons $\cdot \mathrm{m}^{-2} \cdot \mathrm{s}^{-1}$ ) and two others were chilled and the irradiance was simultaneously reduced to $3 \mu \mathrm{mol}$ photons $\cdot \mathrm{m}^{-2} \cdot \mathrm{s}^{-1}$. The remaining two untreated cultures served as controls. Chilling was induced at the end of the dark phase at day 0 , defined as the beginning of the exponential growth phase. The freezing point of seawater was reached approximately $50 \mathrm{~min}$ after temperature reduction and the light phase commenced. Samples were taken during the light phase of day 1 at 1,9 , and $17 \mathrm{~h}$ after chilling. These time points also represented the beginning, middle, and end of the light phase and therefore might indicate circadian rhythm of gene expression. At day 2 and all subsequent days, samples were taken in the middle of the light phase to avoid gene expression patterns that are related to circadian rhythm rather than to temperature and light effects.

Biooptics and biochemistry. In vivo quantum yield of photosynthesis at PSII $\left(\mathrm{F}_{\mathrm{v}} / \mathrm{F}_{\mathrm{m}}\right)$ was determine using a Xenon-PAM Fluorometer (Walz, Effeltwich, Germany) equipped with a stirrer and a cooling device, whereas reoxidation measurements were performed with a dual-modulation LED Kinetic Fluorometer (Trtilek et al. 1997). All fluorescence induction measurements were done on samples that had been dark adapted for $15 \mathrm{~min}$. Consequently, the fluorescence data reveal the potential maximum photosynthetic performance and not the in situ kinetics. Reoxidation kinetics of $Q_{\mathrm{A}}$ were measured by decay of variable fluorescence back to fluorescent level during light induction at the origin state. The decay kinetics reflect the reoxidation of $Q_{A}$ by $Q_{B}$ and can be fitted to a set of equations according to Mock and Kroon (2002). Pigment composition was determined by HPLC as described previously (Mock and Kroon 2002).

Macroarrays. Gene probes for the macroarray analysis were based on an EST library of $F$. cylindrus (unpublished data). Macroarrays were composed of 44 selected genes (Table 1). Gene specific oligonucleotides (50mer) (see supplemental Table 1 at http://www.blackwellpublishing.com/ products / journals / suppmat/jpy/jpy40-4/03224_suppl_table. pdf) were selected and synthesized by Qiagen/Operon (Hilden, Germany) and spotted ( $1 \mu \mathrm{g}$ for each oligo) with a slotblot spotting device (Biorad, Munich, Germany) on positively charged nylon membranes (Amersham, Braunschweig, Germany). Total RNA from several time points of all treatments was isolated using the RNAqueous Kit according to the manufacturers instructions (Ambion, Austin, TX, USA) and used for the labeling reaction. Radioactive labeled cDNA was prepared from the total RNA pool by direct incorporation of dCTP $^{32}$ during the first-strand reverse transcriptase reaction, using the EndoFree RT Kit (Ambion). Reverse transcription was performed at $42^{\circ} \mathrm{C}$ for $1 \mathrm{~h}$ using anchored oligodT and random decamer primers (Ambion). Unincorporated radioactive nucleotides were removed using 200-bp spin columns (Amersham). At least $30 \%$ of the added dCTP ${ }^{32}$ were incorporated. Hybridization was conducted with the ULTRAarray hybridisation buffer according to the manufacturers instruction as well as washing of membranes (Ambion). We used $3 \times 2 \mu \mathrm{g} \pm 50 \mathrm{ng}$ total RNA (measured with the Eppendorf BioPhotometer, Hamburg, Germany) from each batch culture for independent labeling reactions with dCTP $^{32}$. Labeled cDNA was then hybridized to different macroarrays. At least triplicate hybridizations for each batch culture ( = at least 6 for each treatment) were conducted with freshly synthesized ${ }^{32} \mathrm{P}$-labeled cDNA. The macroarrays were scanned with a FujiFilm Fluorescent Image Analyzer FLA3000 (FujiFilm, Hamburg, Germany). Data analyses were performed using Aida software (FujiFilm). The raw data were normalized to the intensity of ribosomal genes $30 \mathrm{~S}$ and 40S. Negative control (Escherichia coli total RNA) and array background were subtracted (see supplemental Table 2 at http://www.blackwellpublishing.com/products/journals / suppmat/jpy/jpy40-4/03224_suppl_table.pdf).

\section{RESULTS}

Developmental acclimation at $-1.8^{\circ} \mathrm{C}$ and $35 \mu \mathrm{mol}$ photons $\cdot m^{-2} \cdot s^{-1}$. Cell division ceased for 4 days 
Table 1. Gene composition of macroarray.

\begin{tabular}{|c|c|c|c|c|c|c|}
\hline & & Gene & NCBI acc. no. & Best match BLASTX & Score & e value \\
\hline A & $\begin{array}{l}1 \\
2 \\
3 \\
4 \\
5 \\
6\end{array}$ & $\begin{array}{l}r b c \mathrm{~L} \\
p s b A \\
\text { psbC } \\
\text { psbU } \\
\text { plastid orf } 148 \\
\text { Gapdh }\end{array}$ & $\begin{array}{l}\text { CF076101 } \\
\text { CF076102 } \\
\text { CF076103 } \\
\text { CF076104 } \\
\text { CF076106 } \\
\text { CF076105 }\end{array}$ & $\begin{array}{l}\text { P49520 } \\
\text { AAM62069 } \\
\text { P49472 } \\
\text { AAO43195 } \\
\text { NP } 043612 \\
\text { AAF } 34329\end{array}$ & $\begin{array}{r}966 \\
335 \\
264 \\
134 \\
69 \\
316\end{array}$ & $\begin{array}{l}0.0 \\
\mathrm{e}-118 \\
2 \mathrm{e}-70 \\
6 \mathrm{e}-31 \\
2 \mathrm{e}-11 \\
2 \mathrm{e}-85\end{array}$ \\
\hline B & $\begin{array}{l}1 \\
2 \\
3 \\
4 \\
5 \\
6\end{array}$ & $\begin{array}{l}f c p 1 \\
f c p 2 \\
\text { fcp } 3 \\
\text { vacuolar sorting receptor } \\
\text { plastid ftsH protease } \\
\text { NADPH flavin oxidoreductase }\end{array}$ & $\begin{array}{l}\text { CF076107 } \\
\text { CF076108 } \\
\text { CF076109 } \\
\text { CF076122 } \\
\text { CF076112 } \\
\text { CF076119 }\end{array}$ & $\begin{array}{l}\text { CAA04401 } \\
\text { CAA04404 } \\
\text { Q08587 } \\
\text { AAB72112 } \\
\text { BAA33755 } \\
\text { AAB91098 }\end{array}$ & $\begin{array}{r}69 \\
130 \\
142 \\
89 \\
187 \\
39\end{array}$ & $\begin{array}{l}7 \mathrm{e}-12 \\
4 \mathrm{e}-30 \\
1 \mathrm{e}-33 \\
2 \mathrm{e}-17 \\
7 \mathrm{e}-47 \\
0.019\end{array}$ \\
\hline C & $\begin{array}{l}1 \\
2 \\
3 \\
4 \\
5 \\
6\end{array}$ & $\begin{array}{l}\text { cytochrome oxidase I } \\
\text { cell division cycle protein } \\
\text { mitochondrial matrix protein precursor } \\
\text { UDP galactose translocase } \\
\text { acetyl-CoA-carboxylase } \\
\text { adenosylsulfatereductase }\end{array}$ & $\begin{array}{l}\text { CF076110 } \\
\text { CF076111 } \\
\text { CF076135 } \\
\text { CF076136 } \\
\text { CF076115 } \\
\text { CF076128 }\end{array}$ & $\begin{array}{l}\text { BBA86610 } \\
\text { AAB40928 } \\
\text { CAA72325 } \\
\text { Q9R0M8 } \\
\text { AAA81471 } \\
\text { AAC26979 }\end{array}$ & $\begin{array}{r}110 \\
84 \\
95 \\
154 \\
129 \\
61\end{array}$ & $\begin{array}{l}9 \mathrm{e}-24 \\
1 \mathrm{e}-15 \\
4 \mathrm{e}-19 \\
4 \mathrm{e}-37 \\
3 \mathrm{e}-34 \\
6 \mathrm{e}-09\end{array}$ \\
\hline $\mathrm{D}$ & $\begin{array}{l}1 \\
2 \\
3 \\
4 \\
5 \\
6\end{array}$ & $\begin{array}{l}\text { delta } 12 \text { desaturase } \\
\text { phosphoglycerate kinase } \\
\text { disease resistant protein } 1 \\
\text { DEAD/DEAH box RNA helicase } \\
\text { cold acclimation protein } 160 \\
\text { disease resistant protein } 2\end{array}$ & $\begin{array}{l}\text { CF076114 } \\
\text { CF076113 } \\
\text { CF076116 } \\
\text { CF076118 } \\
\text { CF076123 } \\
\text { CF076126 }\end{array}$ & $\begin{array}{l}\text { AAO23564 } \\
\text { AAF45020 } \\
\text { AAC78591 } \\
\text { AAF27002 } \\
\text { AAC49998 } \\
\text { NP_187719 }\end{array}$ & $\begin{array}{r}137 \\
293 \\
89 \\
136 \\
41 \\
112\end{array}$ & $\begin{array}{l}5 \mathrm{e}-32 \\
1 \mathrm{e}-78 \\
4 \mathrm{e}-17 \\
8 \mathrm{e}-32 \\
0.006 \\
5 \mathrm{e}-24\end{array}$ \\
\hline $\mathrm{E}$ & $\begin{array}{l}1 \\
2 \\
3 \\
4 \\
5 \\
6\end{array}$ & $\begin{array}{l}\text { calcium dependent protein kinase } \\
\text { hypersensitive induced protein } \\
\text { aconitate hydratase } \\
\text { phosphoprotein phosphatase } \\
\text { phosphodiesterase } \\
\text { phosphoribosylpyrophosphatase }\end{array}$ & $\begin{array}{l}\text { CF076117 } \\
\text { CF076129 } \\
\text { CF076124 } \\
\text { CF076125 } \\
\text { CF076127 } \\
\text { CF076130 }\end{array}$ & $\begin{array}{l}\text { AAD55274 } \\
\text { AAF68390 } \\
\text { P36683 } \\
\text { S69215 } \\
\text { CAA61202 } \\
\text { AAF15359 }\end{array}$ & $\begin{array}{r}50 \\
108 \\
179 \\
43 \\
53 \\
157\end{array}$ & $\begin{array}{l}1 \mathrm{e}-05 \\
7 \mathrm{e}-23 \\
7 \mathrm{e}-45 \\
0.002 \\
2 \mathrm{e}-06 \\
5 \mathrm{e}-38\end{array}$ \\
\hline $\mathrm{F}$ & $\begin{array}{l}1 \\
2 \\
3 \\
4 \\
5 \\
6\end{array}$ & $\begin{array}{l}\text { heat shock protein } 70 \\
\text { pumilio MPT5 } \\
\text { GTPase } \\
\text { oxidoreductase } \\
\text { plastid elongation factor } \\
\text { cytoplasm elongation factor }\end{array}$ & $\begin{array}{l}\text { CF076138 } \\
\text { CF076134 } \\
\text { CF076137 } \\
\text { CF076144 } \\
\text { CF076120 } \\
\text { CF076131 }\end{array}$ & $\begin{array}{l}\text { CAA62478 } \\
\text { AAC95216 } \\
\text { AAC37588 } \\
\text { NP } 334864 \\
\text { O78481 } \\
\text { P16521 }\end{array}$ & $\begin{array}{r}34 \\
158 \\
73 \\
57 \\
44 \\
105\end{array}$ & $\begin{array}{l}0.20 \\
3 \mathrm{e}-38 \\
2 \mathrm{e}-15 \\
1 \mathrm{e}-07 \\
7 \mathrm{e}-04 \\
2 \mathrm{e}-22\end{array}$ \\
\hline $\mathrm{G}$ & $\begin{array}{l}1 \\
2 \\
3 \\
4 \\
5 \\
6\end{array}$ & $\begin{array}{l}\text { calpain protease } \\
\text { ubiquitin activating enzyme } \\
\text { ATP binding protein } \\
\text { ribosomal protein } 30 S \text { pos. control } \\
\text { ribosomal protein } 4 S \\
\text { ribosomal protein } 3 S\end{array}$ & $\begin{array}{l}\text { CF076121 } \\
\text { CF076132 } \\
\text { CF076133 } \\
\text { CF076140 } \\
\text { CF245455 } \\
\text { CF076142 }\end{array}$ & $\begin{array}{l}\text { NP } 033926 \\
\text { O94609 } \\
\text { NP_062425 } \\
\text { NP } 143612 \\
\text { CAA } 58934 \\
\text { AAB } 19349\end{array}$ & $\begin{array}{r}115 \\
162 \\
91 \\
47 \\
41 \\
183\end{array}$ & $\begin{array}{l}3 \mathrm{e}-25 \\
2 \mathrm{e}-39 \\
6 \mathrm{e}-18 \\
2 \mathrm{e}-05 \\
0.038 \\
7 \mathrm{e}-46\end{array}$ \\
\hline $\mathrm{H}$ & $\begin{array}{l}1 \\
2 \\
3\end{array}$ & $\begin{array}{l}\text { ribosomal protein } 40 S \text { pos. control } \\
\text { ribosomal protein } L 2 \\
\text { Escherichia coli total } R N A \text { neg. control }\end{array}$ & $\begin{array}{l}\text { CF076139 } \\
\text { CF076141 }\end{array}$ & $\begin{array}{l}\text { P49154 } \\
\text { P94270 }\end{array}$ & $\begin{array}{l}198 \\
127\end{array}$ & $\begin{array}{l}1 \mathrm{e}-60 \\
4 \mathrm{e}-29\end{array}$ \\
\hline
\end{tabular}

immediately after cold exposure to the freezing point $\left(-1.8^{\circ} \mathrm{C}\right)$ of seawater (Fig. 1). Cold shock photoinhibition as the first photophysiological response after cold exposure was moderate. Electron transport between plastoquinone $A\left(Q_{A}\right)$ and bound $Q_{B}$ was reduced by $30 \%$ and recovered to control values at the end of the first day (Fig. 2a). Photochemical efficiency $\left(\mathrm{F}_{\mathrm{v}} / \mathrm{F}_{\mathrm{m}}\right)$ decreased by only $15 \%$ at day 1 with subsequent recovery within the following 5 days (Fig. $2 b$ ). Interestingly, cellular concentrations of $\operatorname{chl} a$ and $\operatorname{chl} c$ increased (Fig. 3, a and b), as did the ratio of $\operatorname{chl} c / \operatorname{chl} a$, indicating that chl $c$ was more abundant after cold exposure (Fig. 3c). Cellular concentration of fucoxanthin was slightly higher for 2 days after cold exposure compared with the control culture at $+5^{\circ} \mathrm{C}$ (Fig. 3d). Diatoxanthin (DT) cellular concentration increased at least 4-fold compared with the control culture (Fig. $3 \mathrm{e}$, whereas the concentration of diadinoxanthin (DD) remained more or less at control levels (Fig. 3f). Thus, the ratio of DT/DD increased from 1 up to 5 after cold exposure (Fig. 3 g).

Cold exposure induced significant changes in the expression level of certain genes. At least $30 \%$ of genes on the macroarray showed significant changes (Fig. 4), with strongest effects observed for photosynthesis related genes. Steady state mRNA levels of $p s b A$ (Fig. 5a), $p s b U$ (Fig. 5b), psbC (Fig. 4), and three fucoxanthin/chl $a$ binding proteins $(f c p)$ (Figs. 4 , and $5 c$ ) decreased at 
TABLE 2. Gene expression during temperature shifts.

\begin{tabular}{|c|c|c|c|c|}
\hline \multirow[b]{2}{*}{ Gene } & \multicolumn{2}{|c|}{$35 \mu \mathrm{mol}$ photons $\cdot \mathrm{m}^{-2} \cdot \mathrm{s}^{-1}$} & \multicolumn{2}{|c|}{$3 \mu \mathrm{mol}$ photons $\cdot \mathrm{m}^{-2} \cdot \mathrm{s}^{-1}$} \\
\hline & Short term & Long term & Short term & Long term \\
\hline & \multicolumn{4}{|c|}{ Light independent gene expression } \\
\hline Up-regulated & & & & \\
\hline cell division cycle protein & + & ++ & ++ & ++ \\
\hline pumilio $M P T 5$ & + & ++ & ++ & ++ \\
\hline \multicolumn{5}{|l|}{ Down-regulated } \\
\hline$r b c \mathrm{~L}$ & - & - & - & - - \\
\hline$p s b A$ & $-\quad-$ & $-\quad-$ & $-\quad-$ & $-\quad-$ \\
\hline$p s b C$ & - & - & - & - \\
\hline ATP binding protein & - & - & - & - \\
\hline \multicolumn{5}{|l|}{ Short term up-regulated } \\
\hline$D E A D / D E A H$ box $R N A$ helicase & ++ & $\mathrm{O}$ & ++ & $\mathrm{O}$ \\
\hline GTPase & ++ & $\mathrm{O}$ & + & $\mathrm{O}$ \\
\hline hypersensitive induced protein & ++ & $\mathrm{O}$ & ++ & $\mathrm{O}$ \\
\hline \multicolumn{5}{|l|}{ Long term up-regulated } \\
\hline vacuolar sorting receptor & $\mathrm{O}$ & + & $\mathrm{O}$ & + \\
\hline aconitate hydratase & $\mathrm{O}$ & + & $\mathrm{O}$ & + \\
\hline \multicolumn{5}{|l|}{ Long term down-regulated } \\
\hline plastid orf 148 & $\mathrm{O}$ & - & $\mathrm{O}$ & - \\
\hline disease resistant protein 1 & $\mathrm{O}$ & $-\quad-$ & $\mathrm{O}$ & $-\quad-$ \\
\hline calpain protease & $\mathrm{O}$ & - & $\mathrm{O}$ & - \\
\hline ubiquitin activating enzyme & $\mathrm{O}$ & - & $\mathrm{O}$ & - \\
\hline & \multicolumn{4}{|c|}{ Light dependent gene expression } \\
\hline $35 \mu \mathrm{mol}$ photons $\cdot \mathrm{m}^{-2} \cdot \mathrm{s}^{-1}$ up-regulated & & & & \\
\hline plastid ftsH protease & + & ++ & $\mathrm{O}$ & $\mathrm{O}$ \\
\hline mitochondrial matrix protein precursor & ++ & + & $\mathrm{O}$ & $\mathrm{O}$ \\
\hline phosphoribosylpyrophosphatase & ++ & + & $\mathrm{O}$ & - \\
\hline heat shock protein 70 & ++ & + & -- & $-\quad-$ \\
\hline plastid elongation factor & ++ & + & $\mathrm{O}$ & $-\quad-$ \\
\hline cytoplasm elongation factor & + & ++ & - & $\mathrm{O}$ \\
\hline \multirow[t]{2}{*}{ plastid ribosomal protein $4 S$} & \multirow{2}{*}{\multicolumn{4}{|c|}{ Otherwise regulated }} \\
\hline & & & & \\
\hline$p s b U$ & - & + & + & $\mathrm{O}$ \\
\hline$f c p 1$ & $-\quad-$ & $\mathrm{O}$ & $\mathrm{O}$ & $\mathrm{O}$ \\
\hline$f c p 2$ & $\mathrm{O}$ & - & $\mathrm{O}$ & - \\
\hline$f c p 3$ & -- & $\mathrm{O}$ & ++ & $\mathrm{O}$ \\
\hline acetyl-coA-carboxylase & $-\quad-$ & $-\quad-$ & $\mathrm{O}$ & $-\quad-$ \\
\hline disease resistant protein 2 & $\mathrm{O}$ & ++ & $\mathrm{O}$ & $\mathrm{O}$ \\
\hline$G A P D H$ & $\mathrm{O}$ & $\mathrm{O}$ & $\mathrm{O}$ & $\mathrm{O}$ \\
\hline
\end{tabular}

Short term, first day after cold exposure; long term, adapted growth at $-1.8^{\circ} \mathrm{C} ;+$, , 2-fold up-regulated; ++ , $>2$-fold upregulated; -, 2-fold down-regulated; - -, > 2-fold down-regulated; $\mathrm{O}$, no change relative to control culture at $+5^{\circ} \mathrm{C}$ and $35 \mu$ mol photons $\cdot \mathrm{m}^{-2} \cdot \mathrm{s}^{-1}$.

day 1 after cold exposure. The RUBISCO large subunit $(r b c \mathrm{~L})$ gene was also down-regulated (Fig. 5d). In contrast, other genes not primary related to photosynthesis were either transiently up-regulated after cold shock or constantly up-regulated after complete acclimation to freezing temperature. A fast up-regulation after $1 \mathrm{~h}$ or less of cold exposure was observed for $D E A D / D E A H$ box RNA helicase (Fig. 5e), GTPase (Fig. 5f), and plastid translation elongation factor EfTs (Fig. $5 \mathrm{~g})$. Nine hours after cold exposure (i.e. the middle of light phase), a phosphoribosylpyrophosphatese (PRPP) (Fig. $5 \mathrm{~h}$ ), hsp70 (Fig. 5i), plastid ribosomal rpS4 subunit (Fig. 5j), an undefined cell division cycle protein, a gene related to the pumilio family (MPT5) for posttranscription down-regulation, a mitochondrial matrix protein precursor-like protein, and a hypersensitive induced protein were up-regulated (Table 2). At the end of day $1(17 \mathrm{~h})$, some genes remained up-regulated (e.g. $D E A D / D E A H$ box helicase, Fig. 5e) but others were again down-regulated (e.g. hsp70, Fig. 5i). A plastid $f t s H$ protease was about 2-fold up-regulated after cold exposure, and the expression remained more or less constant until the algae started growing again after 4 days of cold treatment (Fig. 5k). An 8-fold up-regulation could be observed for this gene under completely adapted growth, 13 days after cold exposure (long-term regulation, Fig. 5k and Table 2). Longterm up-regulation could also be observed for some other genes (Table 2). In contrast, genes directly involved in photosynthesis $(p s b A, p s b C, r b c \mathrm{~L})$ remained down-regulated even under exponential growth. However, a plastid elongation factor along with a cytoplasmic elongation factor and a plastid ribosomal protein $r p S 4$ remained up-regulated (Table 2).

Developmental acclimation at $-1.8^{\circ} \mathrm{C}$ and 3 umol photons $\cdot m^{-2} \cdot s^{-1}$. Cell division did not immediately cease at freezing temperature and $3 \mu \mathrm{mol}$ photons $\cdot \mathrm{m}^{-2} \cdot \mathrm{s}^{-1}$ (Fig. 1). Cells divided at least once 


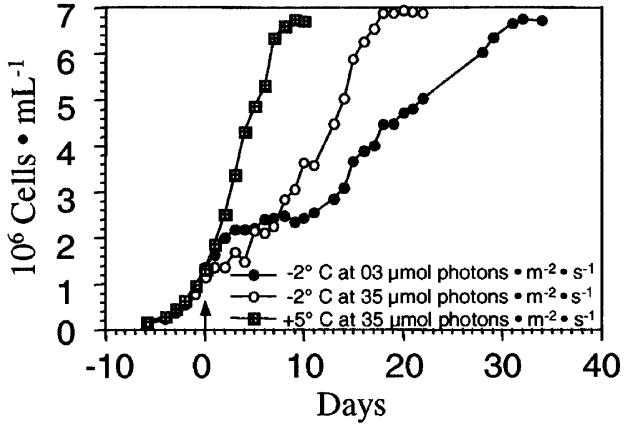

FIg. 1. Temporal development of Fragilariopsis cylindrus cell concentrations. Six cultures were grown at $+5^{\circ} \mathrm{C}$ and $35 \mu \mathrm{mol}$ photons $\cdot \mathrm{m}^{-2} \cdot \mathrm{s}^{-1}$ under a 18:6-h light:dark cycle until day 0 . Four of them were chilled at day 0 (end of dark period) to $-1.8^{\circ} \mathrm{C}$ (arrow), two under unchanged irradiance level (open circles), and two under reduced irradiance level (closed circles). Two cultures (squares) remained unchilled (controls).

more until they ceased their division for 9 days. Cold shock photoinhibition was not initiated by the reduced irradiance. However, a low light acclimation was induced. Quantum yield $\left(\mathrm{F}_{\mathrm{v}} / \mathrm{F}_{\mathrm{m}}\right)$ and electron
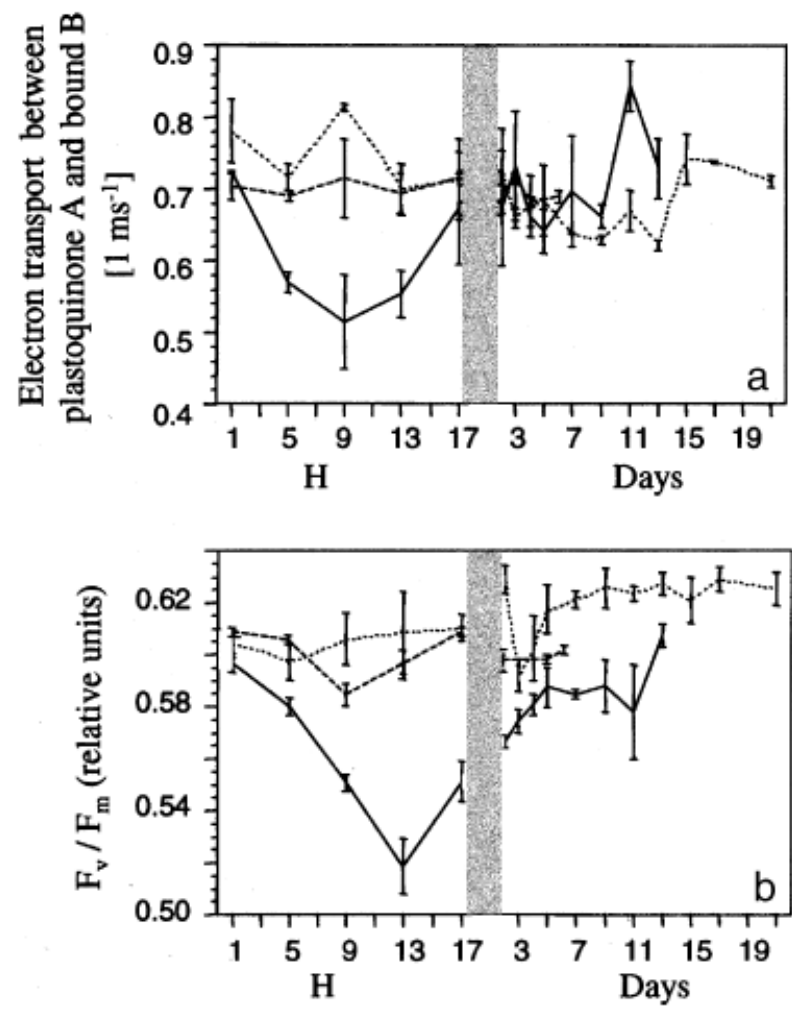

Fig. 2. Temporal development of electron transport between (a) plastoquinone $\mathrm{A}$ and bound $\mathrm{B}$ and (b) quantum yield $\left(\mathrm{F}_{\mathrm{v}} / \mathrm{F}_{\mathrm{m}}\right)$. Measurements at day 1 were conducted on an hourly basis over the light period, whereas measurements were conducted only once a day (mid-light period) on all subsequent days. Solid line represents $-1.8^{\circ} \mathrm{C}$ at $35 \mu \mathrm{mol}$ photons $\cdot \mathrm{m}^{-2} \cdot \mathrm{s}^{-1}$, dotted line $-1.8^{\circ} \mathrm{C}$ at $3 \mu \mathrm{mol}$ photons $\cdot \mathrm{m}^{-2} \cdot \mathrm{s}^{-1}$, and dashed line $+5^{\circ} \mathrm{C}$ at $35 \mu \mathrm{mol}$ photons $\cdot \mathrm{m}^{-2} \cdot \mathrm{s}^{-1}$ (control). $n=10$; error bars, $\pm \mathrm{SD}$.
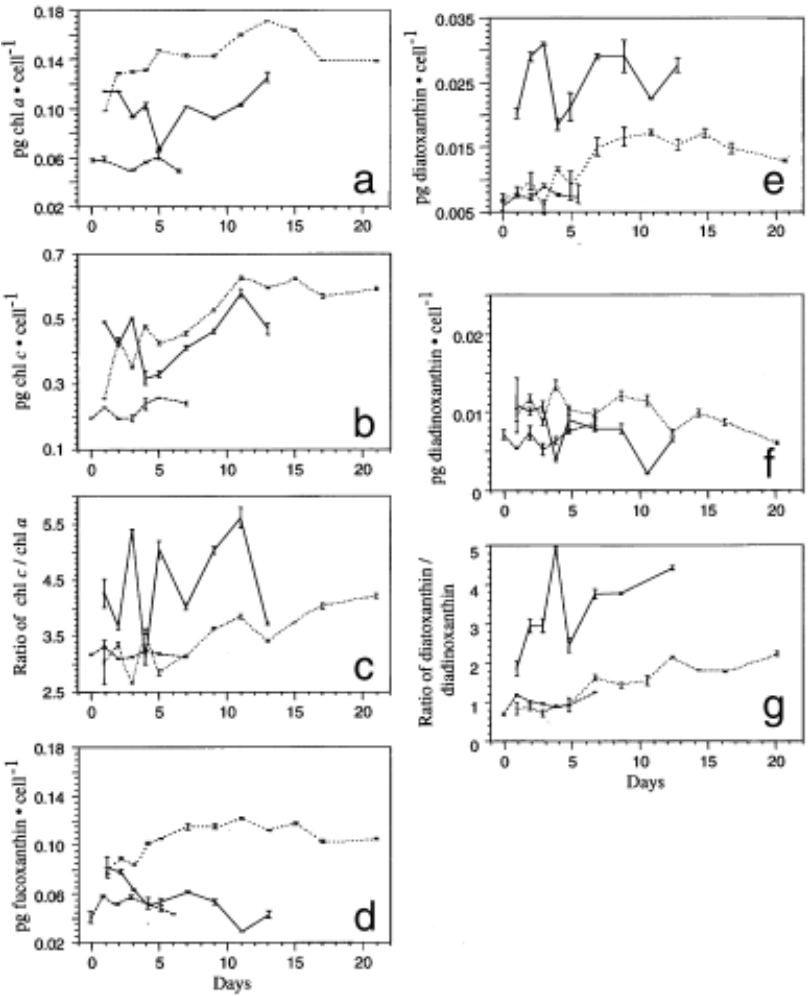

Fig. 3. Temporal development of (a) $\operatorname{chl} a$, (b) $\operatorname{chl} c$, (c) ratio of $\operatorname{chl} c / \operatorname{chl} a$, (d) fucoxanthin, (e) diatoxanthin, (f) diatoxanthin, and $(\mathrm{g})$ ratio of diatoxanthin-to-diadinoxanthin concentrations per cell. Solid line represents $-1.8^{\circ} \mathrm{C}$ at $35 \mu \mathrm{mol}$ photons $\cdot \mathrm{m}^{-2} \cdot \mathrm{s}^{-1}$, dotted line $-1.8^{\circ} \mathrm{C}$ at $3 \mu \mathrm{mol}$ photons $\cdot \mathrm{m}^{-2} \cdot \mathrm{s}^{-1}$, and dashed line $+5^{\circ} \mathrm{C}$ at $35 \mu \mathrm{mol}$ photons $\cdot \mathrm{m}^{-2} \cdot \mathrm{s}^{-1}$ (control). $n=4$; error bars, $\pm \mathrm{SD}$.

transport between $Q_{A}$ and bound $Q_{B}$ remained at the same level as the control $\left(+5^{\circ} \mathrm{C}\right)$ or even increased (Fig. 2). Strongly increasing cellular concentrations of chl $a$ and chl $c$ were measured immediately after cold exposure and reduced irradiance (Fig. 3, a and b). The ratio of chl $c$ to chl $a$ remained at control levels with slightly increasing concentrations at acclimated growth (Fig. 3c). The same was observed for the ratio of DT to DD (Fig. 3g). Marginally increasing cellular DT concentrations at the end of the acclimation phase (approximately day 7 after cold exposure) caused an increased ratio of these pigments. However, the beginning of exponential growth was at day 10 , and the growth proceeded under the new conditions at a rate of $0.22 \mathrm{~d}^{-1}$ (Fig. 1).

Significant changes in gene expression after cold exposure and irradiance reduction could be also detected for photosynthesis related genes. Expression of $p s b A, p s b C$, and $r b c \mathrm{~L}$ was down-regulated by the same order of magnitude compared with cold exposure without reduction of irradiance (Fig. 5, Table 2). In contrast, $p s b U$ and two $f c p$ genes were up-regulated after cold exposure at reduced light (Fig. 5, b and c, Table 2). Fast up-regulation ( $1 \mathrm{~h}$ after changes) could also be observed for the $D E A D / D E A H$ box helicase 


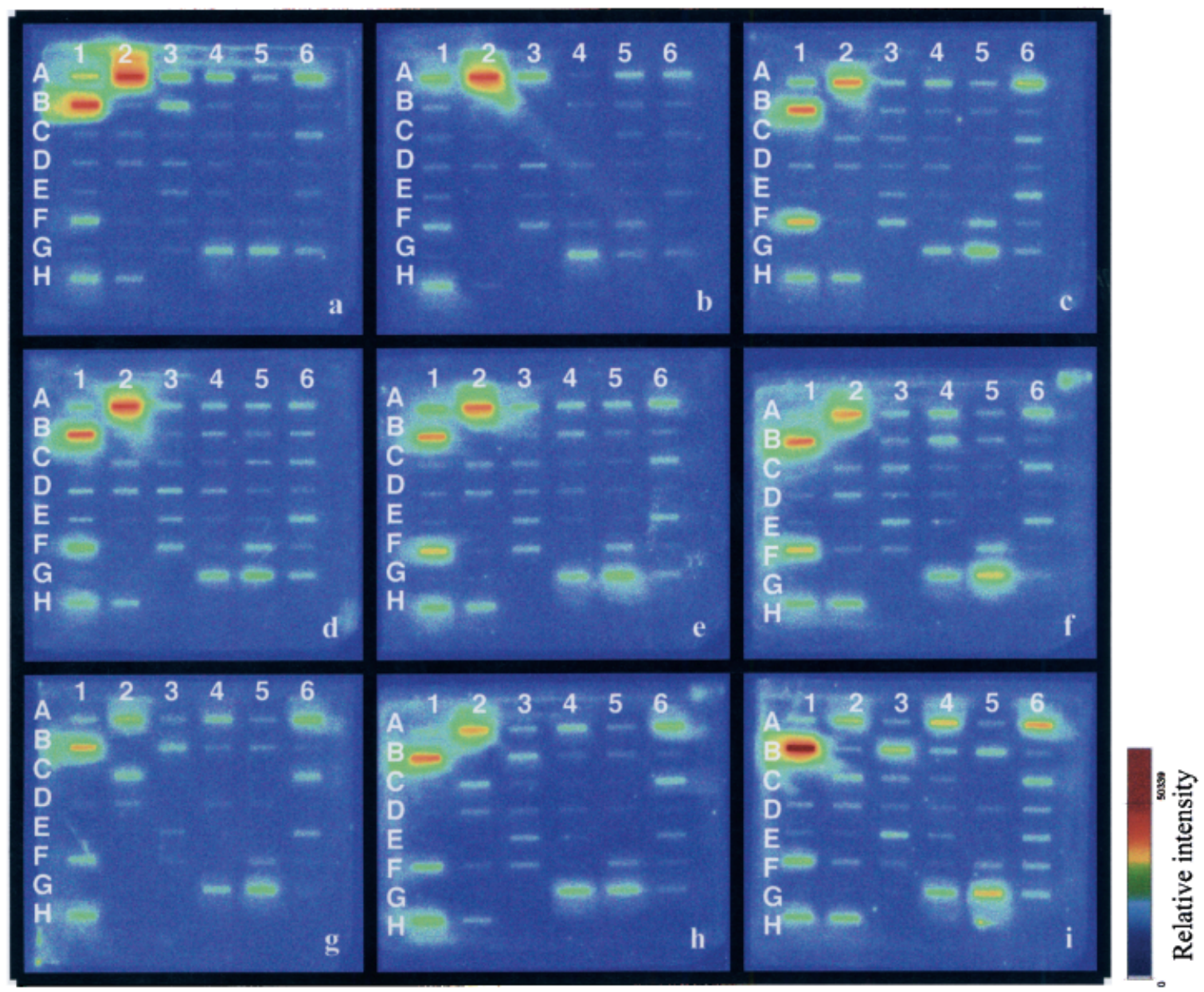

Fig. 4. Macroarrays of which each is composed of 44 genes (Table 1) spotted as oligonucleotides (50mer) in horizontal lines. (A) $+5^{\circ} \mathrm{C}$ at $35 \mu \mathrm{mol}$ photons $\cdot \mathrm{m}^{-2} \cdot \mathrm{s}^{-1}$ (middle of light period, $9 \mathrm{~h} \mathrm{light)}$. (B-I) $-1.8^{\circ} \mathrm{C}$ at $35 \mu \mathrm{mol}$ photons $\cdot \mathrm{m}^{-2} \cdot \mathrm{s}^{-1}$. (B) $1 \mathrm{~h},(\mathrm{C}) 9 \mathrm{~h},(\mathrm{D}) 17 \mathrm{~h}$ after cold exposure on day 1. (E) Day 2, (F) day 3, (G) day 4, (H) day 5, (I) day 13 after cold exposure measured in the middle of light period, $9 \mathrm{~h}$ light.

(Fig. 5e), GTPase (Fig. 5f), and plastid translation elongation factor EfTS (Fig. $5 \mathrm{~g}$ ). Nine hours later the $D E A D / D E A H$ box helicase was still up-regulated, whereas the other genes were again down-regulated. However, the undefined cell division cycle protein, the pumilio family (MPT5) related gene, and the hypersensitive induced protein were also up-regulated in the same order of magnitude compared with $35 \mu \mathrm{mol}$ photons $\cdot \mathrm{m}^{-2} \cdot \mathrm{s}^{-1}$ (Table 2). In contrast to results at 35 $\mu \mathrm{mol}$ photons $\cdot \mathrm{m}^{-2} \cdot \mathrm{s}^{-1}$, some genes remained at control levels during short- and long-term acclimation at 3 $\mu \mathrm{mol}$ photons $\cdot \mathrm{m}^{-2} \cdot \mathrm{s}^{-1}$ and freezing temperature (e.g. PRPP, rpS4, plastid ftsH protease, Fig. 5) or were even down-regulated (e.g. hsp70).

\section{DISCUSSION}

The comparison between results at 35 and $3 \mu \mathrm{mol}$ photons $\cdot \mathrm{m}^{-2} \cdot \mathrm{s}^{-1}$ at freezing temperatures identifies genes clusters that are regulated in a light independent fashion, a light dependent way, and otherwise regulat- ed (Table 2). Furthermore, we could differentiate between genes that were short-term or long-term regulated under both light conditions after cold exposure.

Some genes important for photosynthesis $(r b c \mathrm{~L}$, $p s b A, p s b C$ ) were down-regulated in a light independent way even after recovery of cell division. Thus, low temperature has an overall negative effect on transcription of these genes in F. cylindrus. These genes are crucial for photosynthetic electron transport ( $p s b C$, $p s b A)$ and $\mathrm{CO}_{2}$ fixation $(r b c \mathrm{~L})$ and therefore for growth after the acclimation phase. Growth proceeded but the concentration of $r b c \mathrm{~L}$ mRNA remained low. However, the demand for organic carbon should be higher under exponential growth than under stationary growth. A posttranscriptional up-regulation of $r b c \mathrm{~L}, p s b A$, and $p s b C$ is conceivable but still unknown for diatoms. Cellular chl $c$ concentrations were higher at freezing temperatures and showed a further slight increase at higher irradiances. Chl $c$ is a major diatom pigment with absorbance maxima for blue-green light and 

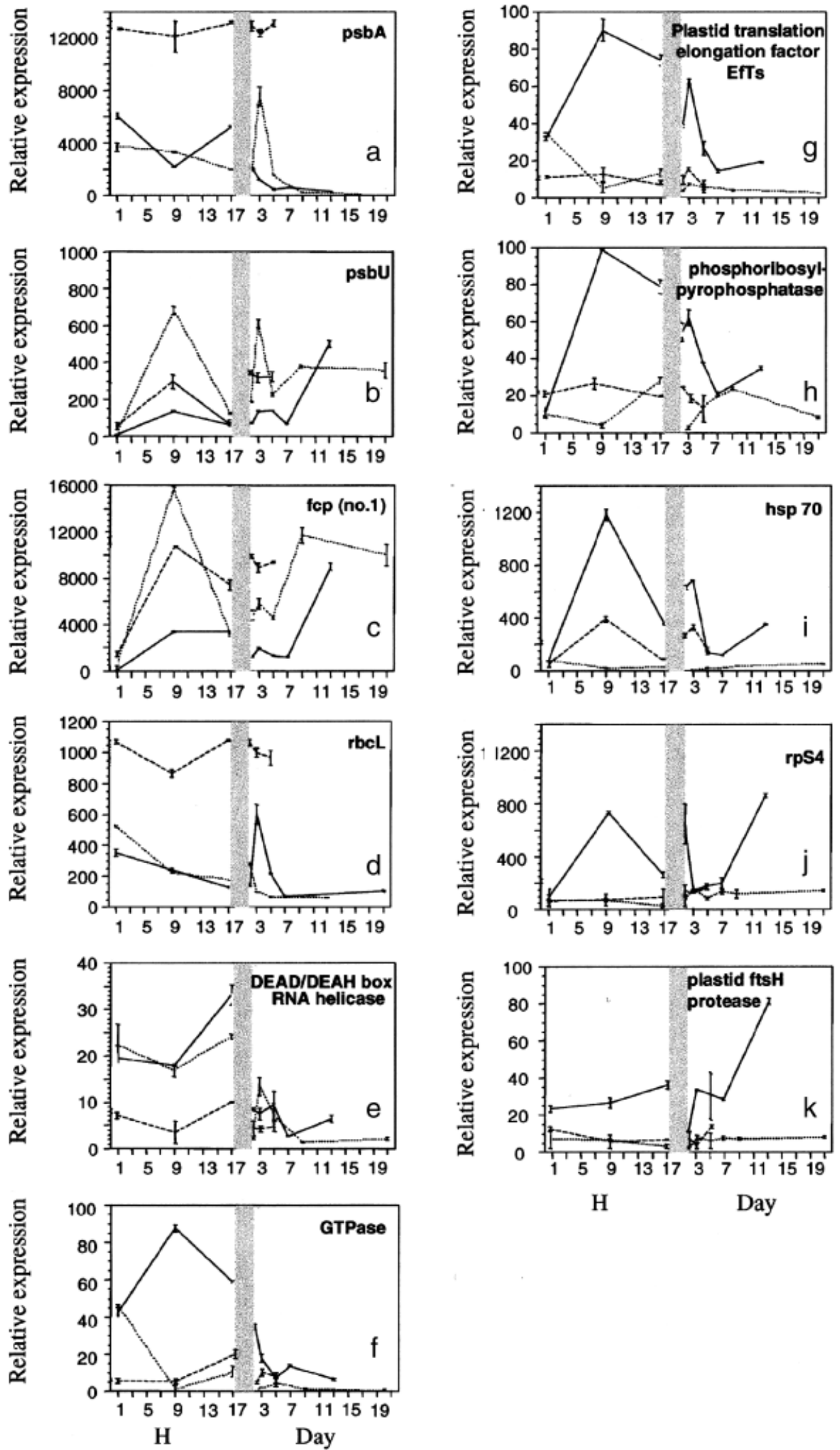

$\mathrm{H}$

Day

FIG. 5. Temporal expression of selected genes from the first hour after cold exposure to a completely acclimated state. ((a) psbA, (b) psbU, (c) $f c p(n o .1)$, (d) rbcL, (e) DEAD/DEAH, (f) GTPase, (g) EfTs, (h) phosophribosylpyrophosphatase, (i) hsp70, (j) rpS4, (k) ftsH). Solid line represents $-1.8^{\circ} \mathrm{C}$ at $35 \mu \mathrm{mol}$ photons $\cdot \mathrm{m}^{-2} \cdot \mathrm{s}^{-1}$, dotted line $-1.8^{\circ} \mathrm{C}$ at $3 \mu \mathrm{mol}$ photons $\cdot \mathrm{m}^{-2} \cdot \mathrm{s}^{-1}$, and dashed line $+5^{\circ} \mathrm{C}$ at $35 \mu \mathrm{mol}$ photons $\cdot \mathrm{m}^{-2} \cdot \mathrm{s}^{-1}$ (control). $n=6$; error bars $= \pm \mathrm{SD}$. 
therefore essential in aquatic environments where the penetration depth of red light is reduced, particularly under sea ice (Boczar and Palmisano 1990, Falkowski and LaRoche 1991). However, chl $c$ must have an additional function regarding low-temperature acclimation, which could be related to a reorganization of light-harvesting complexes (Boczar and Palmisano 1990). Interestingly, cellular chl $a$ concentrations also were slightly higher after cold exposure and higher light intensity $\left(35 \mu \mathrm{mol}\right.$ photons $\left.\cdot \mathrm{m}^{-2} \cdot \mathrm{s}^{-1}\right)$, a condition that induced light stress in F. cylindrus as indicated by decreasing $\mathrm{F}_{\mathrm{v}} / \mathrm{F}_{\mathrm{m}}$ and decreasing electron transport rates. Cellular DT concentrations were up-regulated, possibly to protect the photosystems, and two fcps (nos.1 and 3) were down-regulated over the short term, despite increased chl $a$ concentrations, which is therefore contrary to what we know from green algae regarding interactions between light and temperature (Maxwell et al. 1994, 1995). However, there are usually more than three different fcps in diatoms (Eppard and Rhiel 2000), and some of them are up-regulated under higher irradiances (Oeltjen et al. 2002).

In contrast, some genes were also short- and longterm up-regulated under this light-stress condition (light dependent gene expression, Table 2). These were three plastid localized and two cytoplasmic proteins related to protein synthesis (translation, elongation factors, ribosomal protein $r p S 4, P R P P)$, protein turnover ( $f t s H$ protease), and the chaperon $h s p 70$. Upregulation of these genes provides evidence that there was an enhancement of translation at $-1.8^{\circ} \mathrm{C}$ and $35 \mu \mathrm{mol}$ photons $\cdot \mathrm{m}^{-2} \cdot \mathrm{s}^{-1}$ compared with $-1.8^{\circ} \mathrm{C}$ and $3 \mu \mathrm{mol}$ photons $\cdot \mathrm{m}^{-2} \cdot \mathrm{s}^{-1}$. Enhanced protein synthesis could be necessary to provide new proteins for repair of photodamaged proteins in the thylakoid membrane, such as reaction center protein of PSII. Strong long-term up-regulation of the plastid $\mathrm{fts} H$-protease at acclimated growth indicated increased turnover of the reaction center protein of PSII, which is the main target protein for this protease in higher plants (Adam and Clarke 2002). The $\mathrm{fts} H$-protease as well as the other light-dependent regulated genes were not up-regulated at freezing temperatures and low irradiance, which supports the hypothesis that temperature acclimation in F. cylindrus is related to repair of photodamaged proteins even at irradiances that are not per se inhibiting to photosynthesis and are typical for lowlight polar environments (Thomas and Dieckmann 2002). When low-light acclimated diatoms are exposed to higher irradiances or to lower temperature, which mimics higher irradiances (Maxwell et al. 1994), a nonphotochemical quenching is rapidly developed (Olaizola et al. 1994). Non-photochemical quenching is associated with a xanthophyll cycle (DD cycle), which differs from that of higher plants. The DD cycle converts the monoepoxide carotenoid DD into the deepoxide form DT under high light and DT back to DD under low light or in darkness (Arsalane et al. 1994, Lavaud et al. 2002). However, even at $3 \mu \mathrm{mol}$ photons $\cdot \mathrm{m}^{-2} \cdot \mathrm{s}^{-1}$, the cellular DT concentrations in- creased, possibly for protection against light. Under such low light conditions, this physiological process is probably sufficient for protection, making additional repair processes unnecessary.

In contrast to photosynthesis related genes, two genes for mitochondrial respiration on the array (cytochrome oxidase I and mitochondrial matrix protein precursor) gave no clear trends for a higher or lower demand for proteins involved in respiration. However, two other genes related to degradation of proteins (calpain protease and ubiquitin activating enzyme) were long-term down-regulated after cold exposure, independently of irradiance (Table 2). This fact gives some evidence for a possible reduction of catabolic processes at freezing temperatures, which has already been proposed for cold living algae (Raven et al. 2002).

We could also identify genes not primarily involved in photosynthesis and respiration but possibly involved in acclimation to low temperatures, which is indicated by their strong short- and long-term up-regulation after cold exposure regardless of irradiance. Two of these rapidly up-regulated genes are a $D E A D / D E A H$ box RNA helicase and a GTPase without a known specific function. RNA helicases catalyze the unwinding of RNA duplexes and secondary structures in an ATPdependent manner (De la Cruz et al. 1999). The formation of RNA duplexes and secondary structures such as stem-loops and hairpins are more likely at low temperatures. Enhanced reorganization of mRNAs by $D E A D / D E A H$ box RNA helicases, of which some also have chaperon functions, possibly could keep transcription active after cold exposure in F. cylindrus. An unspecified hypersensitive induced protein was also short-term up-regulated after cold shock in F. cylindrus, whereas a vacuolar sorting receptor as well as an aconitate hydratase, known as a cold shock gene in Bacillus subtilis (Lottering and Streips 1995), were up-regulated only under completely acclimated growth. Vacuolar sorting receptors select proteins at the Golgi apparatus for sorting to clathrin-coated vesicles and delivery to the vacuole (Kirsch et al. 1994). Selection of new proteins, which were probably produced during the acclimation phase and their transport to prevacuoles were thus induced. Osmoregulatory substances (e.g. prolin) are often stored in vacuoles to avoid dehydration caused by increasing salinities in brine during freezing of seawater (Xiong and Zhu 2002). Diatoms have a large central vacuole where osmoregulatory substances could be stored.

A cell division cycle related gene was permanently up-regulated under both irradiances. Because cell division actually was reduced, this up-regulation probably had a negative effect on cell division. This regulation principle is known for many proteins involved in the cell division cycle (Dewitte and Murray 2003). Another possibly negatively and light independently regulated gene after cold exposure belongs to the pumilio MPT5 family (Table 2). Proteins from this family serve as multicopy suppressors of translation, which could 
mean that some genes in F. cylindrus were also posttranscriptional down-regulated under freezing temperatures. Studies with MPT5 mutants showed that they exhibit many defects, including temperature sensitive growth (Hata et al. 1998). Posttranscriptional down-regulation of certain, still unknown, genes in F. cylindrus could therefore be essential for growth under freezing temperatures. The delta12 desaturase (desA) and the cold-stress gene CAP160 were not up-regulated upon the downshift in temperature, but this is regularly measured in temperate photoautotrophes (Kaye et al. 1998, Los and Murata 1998), which indicated a different mechanism of gene-expression regulation for both genes in the psychrophilic diatom F. cylindrus.

\section{CONCLUSIONS}

This macroarray study with an ecologically relevant polar diatom provides first insights into how selected genes for photosynthesis, respiration, and cold acclimation are regulated during a temperature reduction to the freezing point of seawater, which occurs each autumn in polar seas. The expression of genes for PSII $(p s b A, p s b C)$ and carbon fixation $(r b c \mathrm{~L})$ was down-regulated. A long-term increased production of the light protection pigment DT, even at $3 \mu$ mol photons $\cdot \mathrm{m}^{-2} \cdot \mathrm{s}^{-1}$, could be observed in F. cylindrus. Chl $c$ concentrations were also higher at lower temperatures. Genes for plastid protein synthesis (translation elongation factor EfTs, ribosomal protein $r p S 4$ ) and turnover of thylakoid-membrane integrated proteins ( $f t s H$ protease) were up-regulated at $35 \mu \mathrm{mol}$ photons $\cdot \mathrm{m}^{-2} \cdot \mathrm{s}^{-1}$ and freezing temperatures, which could indicate that repair of photodamaged proteins occurred at higher irradiances. Down-regulation of PSII genes, $r b c \mathrm{~L}$, DT production, and repair of photodamaged proteins are therefore possibly important mechanisms to keep photosynthesis active in F. cylindrus at freezing temperatures.

Cold acclimation in F. cylindrus seems to be generally related to unwinding of RNA duplexes and secondary structures of RNA, which more easily form at freezing temperatures and which might interfere with protein translation (Mohr et al. 2002). This basic regulatory process was activated by an up-regulation of $D E A D /$ $D E A H$ box RNA helicase. Also, deactivation of translation for certain genes may be of importance under freezing temperatures. Evidence for this was given by a permanently up-regulated pumilio MPT5 gene, which is a multicopy suppressor for gene translation. However, we could not identify which genes were posttranscriptionally suppressed by this enzyme. These genes, as well as other genes related to cold acclimation, remain to be identified to improve our understanding of acclimation of diatoms to the polar environment.

We thank Linda Medlin for providing laboratory space and generous support and Gerhard Dieckmann, Niko Hoch, Erika Allhusen, and Andreas Krell for their helpful collaboration in the laboratory.
Adam, Z. \& Clarke, A. K. 2002. Cutting edge of chloroplast proteolysis. Trends Plant Sci. 7:451-6.

Arsalane, W., Rousseau, B. \& Duval, J.-C. 1994. Influence of the pool size of the xanthophyll cycle on the effects of light stress in a diatom: competition between photoprotection and photoinhibition. Photochem. Photobiol. 60:237-43.

Boczar, B. A. \& Palmisano, A. C. 1990. Photosynthetic pigments and pigment-proteins in natural populations of Antarctic sea ice diatoms. Phycologia 29:470-7.

Booth, J. A. 1984. The epontic algal community of the ice edge zone and its significance to the Davis Strait ecosystem. Arctic $37: 234-43$.

Boyd, P. W. 2002. Environmental factors controlling phytoplankton processes in the Southern Ocean. J. Phycol. 38:844-61.

De la Cruz, J., Kressler, D. \& Linder, P. 1999. Unwinding RNA in Saccharomyces cerevisae: DEAD-box proteins and related families. Trends Biochem. Sci. 24:192-8.

Dewitte, W. \& Murray, J. A. 2003. The plant cell cycle. Annu. Rev. Plant Biol. 54:235-64.

Dufresne, A., Salanoubat, M., Partensky, F., Artiguenave, F., Axmann, I. M., Barbe, V., Duprat, S., Galperin, M. Y., Koonin, E. V., LeGall, F., Makarova, K. S., Ostrowski, M., Oztas, S., Robert, C., Rogozin, I. B., Scanlan, D. J., Tandeau de Marsac, N., Weissenbach, J., Wincker, P., Wolf, Y., Hess, W. R. 2003. Genome sequence of the cyanobacterium Prochlorococcus marinus SS120, a nearly minimal oxyphototrophic genome. Proc. Natl. Acad. Sci. USA 100:10020-5.

Eppard, M. \& Rhiel, E. 2000. Investigations on gene copy number, introns and chromosomal arrangement of genes encoding the fucoxanthin chlorophyll a/c-binding proteins of the centric diatom Cyclotella cryptica. Protist 151:27-39.

Falkowski, P. G. \& LaRoche, J. 1991. Adaptation to spectral irradiance in unicellular algae. J. Phycol. 27:8-14.

Fiala, M. \& Oriol, L. 1990. Light-temperature interactions on the growth of Antarctic diatoms. Polar Biol. 10:629-36.

Fuhrman, J. 2003. Genome sequences from the sea. Nature 424:1001-2.

Gleitz, M., Bartsch, A., Dieckmann, G. S. \& Eicken, H. 1998. Composition and succession of sea ice diatom assemblages in the eastern and southern Weddell Sea, Antarctica. In Lizotte, M. P. \& Arrigo, K. R. [Eds.] Antarctic Sea Ice, Biological Processes, Interactions and Variability. American Geophysical Union, Washington, DC, pp. 107-120.

Gombos, Z., Wada, H. \& Murata, N. 1994. The recovery of photosynthesis from low-temperature photoinhibition is accelerated by the unsaturation of membrane lipids: a mechanism of chilling tolerance. Proc. Natl. Acad. Sci. USA 91: 8787-91.

Grunow, A. 1884. Diatomeen von Franz-Josef Land. Denk. Akad. Wien 48:1-394.

Guillard, R. R. \& Ryther, J. H. 1962. Studies of marine plankton diatoms. I. Cyclotella nana (Husted) and Detonula confervacea (Cleve). Can. J. Microbiol. 8:229-39.

Halldal, P. 1953. Phytoplankton investigations from the weather ship $M$ in the Norwegian Sea, 1948-49. (Including observations during the "Armauer Hansen" cruise, July 1994.). Hvalradets Skr. 38:1-91.

Hata, H., Mitsui, H., Liu, H., Bai, Y., Denis, C. L., Shimizu, Y. \& Sakai, A. 1998. Dhhlp, a putative RNA helicase, associates with the general transcription factors Pop2p and Ccr4p from Saccharomyces cerevisae. Genetics 148:571-9.

Hoag, H. 2003. Genomes take pole position in icy wastes. Nature 421:880.

Jeong, S. W., Choi, S. M., Lee, D. S., Ahn, S. N., Hur, Y., Chow, W. S. \& Park, Y. 2002. Differential susceptibility of photosynthesis to light-chilling stress in rice (Oryza sativa l.) depends on the capacity for photochemical dissipation of light. Mol. Cells 13:419-28.

Kaye, C., Neven, L., Hofig, A., Li, Q.-B, Haskell, D. \& Guy, C. 1998. Characterization of a gene for spinach CAP160 and expression of two spinach cold-acclimation proteins in tobacco. Plant Physiol. 116:1367-77. 
Kirsch, T., Paris, N., Butler, J. M., Beevers, L. \& Rogers, J. C. 1994. Purification and initial characterization of a potential plant vacuolar targeting receptor. Proc. Natl. Acad. Sci. USA 91: 3403-7.

Kirst, G. O. \& Wiencke, C. 1995. Ecophysiology of polar algae. J. Phycol. 31:181-99.

Lavaud, J., Rousseau, B., van Gorkom, H. J. \& Etienne, A.-L. 2002. Influence of the diadinoxanthin pool size on photoprotection in the marine planktonic diatom Phaeodactylum tricornutum. Plant Physiol. 129:1398-406.

Los, D. A. \& Murata, N. 1998. Structure and expression of fatty acid desaturases. Biochim. Biophys. Acta 1394:3-15.

Lottering, E. A. \& Streips, U. N. 1995. Induction of cold shock proteins in Bacillus subtilis. Curr. Microbiol. 30:193-9.

Lyons, J. M. 1973. Chilling injury in plants. Annu. Rev. Plant Physiol. 24:445-66.

Maxwell, D. P., Falk, S., Trick, C. G. \& Huner, N. P. A. 1994. Growth at low temperature mimics high-light acclimation in Chlorella vulgaris. Plant Physiol. 105:535-43.

Maxwell, D. P., Laudenbach, D. E. \& Huner, N. P. A. 1995. Redox regulation of light-harvesting complex II and $c a b$ mRNA abundance in Dunaliella salina. Plant Physiol. 109:787-95.

Mock, T. \& Gradinger, R. 1999. Determination of Arctic ice algal production with a new in situ incubation technique. Mar. Ecol. Prog. Ser. 177:15-26.

Mock, T. \& Kroon, B. M. A. 2002. Photosynthetic energy conversion under extreme conditions. I. Important role of lipids as structural modulators and energy sink under N-limited growth in Antarctic sea ice diatoms. Phytochemistry 61:41-51.

Mohr, S., Stryker, J. M. \& Lambowitz, A. M. 2002. A DEAD-box protein functions as an ATP-dependent RNA chaperone in group I intron splicing. Cell 109:769-79.

Morgan-Kiss, R., Ivanov, A. G. \& Huner, N. P. A. 2002a. The Antarctic psychrophile, Chlamydomonas subcaudata, is deficient in state I-state II transitions. Planta 214:435-45.

Morgan-Kiss, R., Ivanov, A. G., Williams, J., Khan, M. \& Huner, N. P. A. 2002b. Differential thermal effects on the energy distribution between photosystem II and photosystem I in thy- lakoid membranes of a psychrophilic and a mesophilic alga. Biochem. Biophys. Acta 1561:251-65.

Oeltjen, A., Krumbein, W. E. \& Rhiel, E. 2002. Investigations on transcript sizes, steady state mRNA concentrations and diurnal expression of genes encoding fucoxanthin chlorophyll a/c light harvesting polypeptides in the centric diatom Cyclotella cryptica. Plant Biol. 4:250-7.

Okolodkov, Y. B. 1992. Cryopelagic flora of the Chukchi, East Siberian and Laptev Seas. Proc. Nat. Inst. Polar Res. Symp. Polar Biol. Tokyo 5:28-43.

Olaizola, M., Laroche, J., Kolber, Z. \& Falkowski, P. G. 1994. Nonphotochemical fluorescence quenching and the diadinoxanthin cycle in a marine diatom. Photosynth. Res. 41:357-70.

Paasche, E. 1960. Phytoplankton distribution in the Norwegian Sea in June, 1954, related to hydrography and compared with primary production data. Fisk. Skr. Havund. 12:1-77.

Raven, J., Johnston, A. M., Kubler, J. E., Korb, R., McInroy, S. G., Handley, L. L., Scrimgeour, C. M., Walker, D. I., Beardall, J., Clayton, M. N., Vanderklift, M., Fredriksen, S. \& Dunton, K. H. 2002. Seaweeds in cold seas: evolution and carbon acquisition. Ann. Bot. 90:525-36.

Rocap, G., Larimer, F. W., Lamerdin, J., Malfatti, S., Chain, P., Ahlgren, N. A., Arellano, A., Coleman, M., Hauser, L., Hess, W. R., Johnson, Z. I., Land, M., Lindell, D., Post, A. F., Regala, W., Shah, M., Shaw, S. L., Steglich, C., Sullivan, M. B, Ting, C. S., Tolonen, A., Webb, E. A., Zinser, E. R., Chisholm, S. W. 2003. Genome divergence in two Prochlorococcus ecotypes reflects oceanic niche differentiation. Nature 424:1042-7.

Thomas, D. N. \& Dieckmann, G. S. 2002. Antarctic sea ice-a habitat for extremophiles. Science 295:641-4.

Trtilek, M., Kramer, D. M., Koblizek, M. \& Nedbal, L. 1997. Dual-modulation LED kinetic fluorometer. J. Lumin. 72: 597-9.

von Quillfeldt, C. H. 1997. Distribution of diatoms in the Northeast Water Polynya, Greenland. J. Mar. Syst. 10:211-40.

Xiong, L. \& Zhu, J. K. 2002. Molecular and genetic aspects of plant responses to osmotic stress. Plant Cell Environ. 25: 131-9. 